ANÁLISIS

\title{
Impacto social y económico de la violencia en las Américas
}

\author{
Alberto Concha \\ Organización Panamericana de la Salud, \\ Organización Mundial de la Salud, Washington, DC., EE.UU.
}

La violencia es un problema social y de salud pública que ha crecido en las Américas en las últimas décadas con efectos negativos en lo social, la salud y la economía de países, comunidades, familias e individuos. Más de 115.000 personas mueren asesinadas cada año, la mayoría de ellos son hombres; 55,000 cometen suicidio; en 20 a $60 \%$ de los hogares se ejerce alguna forma de violencia intrafamiliar contra niñas, niños, mujeres y ancianos; las pandillas juveniles, involucradas en actividades violentas y criminales, crecen en forma alarmante. Otras formas de violencia son las guerras y los conflictos internos o internacionales, la violencia política, los secuestros, los linchamientos, la violencia multinacional por el crimen organizado y las mafias con su tráfico de narcóticos, el comercio sexual y el contrabando de armas que dificulta el control de la distribución de las mismas. El enfoque de salud pública se basa en un método de trabajo sintetizado así: 1) caracterizar el problema en sus variables básicas de persona, lugar, tiempo, circunstancias y situaciones conexas; 2) identificar causas, asociaciones o factores de riesgo; 3) proponer intervenciones y evaluarlas, y 4) extender las intervenciones. La violencia es un acto intencional de origen multicausal. Diversos factores se interconectan y muestran las relaciones entre ellos. Se comentan los que han sido mayormente estudiados o han mostrado mayor evidencia. Se propone una mirada histórica que integre la represión y el control, la prevención y la recuperación del tejido social. La demanda de gastos por violencia representa una proporción significativa del PIB, estimado en US\$168.000 millones para Latinoamérica. La violencia se puede prevenir. Con proyectos de prevención primaria se busca evitar que ocurra el hecho violento, pero si el evento violento ya ha ocurrido, hay que evitar su repetición o que sea de mayor gravedad, con prevención secundaria. La prevención terciaria se aplica para evitar mayores daños, se dirige a mejorar la calidad de vida. Los programas deben ser integrales y sostenidos en mediano y largo plazo. Finalmente, se comentan las políticas de OPS y OMS y, en especial, el Informe mundial sobre la violencia y la salud, una herramientas de trabajo para la prevención de la violencia.

Palabras clave: violencia, multicausalidad, magnitud, impacto socioeconómico, prevención.

\section{Social and economic impact of violence in the Americas}

Violence is a social and a public health problem that has grown in the Americas in recent decades that has negative effects on social, health, and the economy of countries, communities, families, and individuals. More than 115,000 people are murdered every year, the majority of them are men; other 55,000 commit suicide. In 20 to $60 \%$ of households some form of domestic violence against girls, boys, women, and the elderly occurs; juvenile gangs, involved in violent and criminal activities, increases at alarming rate. Other forms of violence are wars and internal or international conflicts, political violence, abductions, lynching, multinational violence by organized crime units involved in narcotic trafficking, sexual trade or smuggling of weapons. The public health approach is based on a methodology of work, as follows: 1 . Characterization of the problem in its basic variables of person, place, time, circumstances, and related situations; 2. Identification of causes, associations, or risk factors; 3. Proposal of interventions and their evaluation; 4 . Extension of the evaluated interventions. Violence is an intentional act of multicausal origin. Various factors are interconnected showing the relations among them. Those which have been primarily studied or that have shown greater evidence are discussed. A historical look is proposed, that integrates the repression and control, the prevention and the recovery of the social fabric. Violence demands an expenditure of money that represents a significant proportion 
of the GDP that was estimated in $\$ 168$ billion dollars for Latin America. Violence can be prevented. Primary prevention projects are driven to avoid the occurrence of a violent act, but if it has already occurred it is necessary to avoid its repetition, through secondary prevention projects. Tertiary prevention is applied in order to avoid major damages; it aims at improving the quality of life of those already traumatized. The programs should be comprehensive and sustained in the medium and long terms. Finally, comments have been made on PAHO and WHO policies, in particular the recently launched World Report on Violence and Health, a working tool for violence prevention.

Key words: violence, multicausality, magnitude, social and economic impact, prevention.

"Todos estamos perdiendo con la violencia y en este sentido, como algunas epidemias letales, está más allá de las clases y los partidos, cobra víctimas en todas partes, a todos quita la seguridad, a todos priva de una verdadera patria, una patria para pensar y amar, para trabajar, para luchar por los propios intereses para construir un futuro a los hijos, en fin, para reconocerse en ella".

Estanislao Zuleta

\section{Presentación}

La violencia es un problema social y de salud pública que ha crecido en las Américas en las últimas décadas produciendo efectos negativos en lo social, la salud y la economía de países, comunidades, familias e individuos. En este análisis pretendo mostrar no sólo la validez de la afirmación anterior, sino también invitarles a reflexionar sobre la gravedad del fenómeno social y de salud que la violencia representa y, más aún, con el convencimiento que la violencia es prevenible, evitable. No estamos condenados a convivir pasivamente con la violencia, sino debemos comprometernos con la búsqueda de soluciones de corto, mediano y largo plazo. Tal es el compromiso y la visión de la Organización Panamericana de la Salud (OPS) $(1,2)$.

\section{Marco conceptual}

Desde la perspectiva de salud pública, la violencia es un problema porque: 1) produce una alta carga de mortalidad y morbilidad evitable, que afecta especialmente a los niños y niñas, las mujeres y los jóvenes; 2) requiere un elevado valor en la atención médica y, en ocasiones, distorsiona la atención de otros pacientes por la urgencia que

\footnotetext{
$\overline{\text { Correspondencia: }}$

Alberto Concha

conchaal@paho.org

Recibido:25/10/02; aceptado: 20/11/02
}

demandan las víctimas de la violencia; 3) afecta no sólo a la víctima sino también a la familia y su entorno, con efectos negativos inmediatos en lo económico, social y psicológico y, también, con impactos de mediano y largo plazo; 4) afecta de manera negativa el desarrollo social y económico de comunidades y países, y 5) afecta la cotidianidad de la comunidad, el derecho a disfrutar, a movilizarse, a hacer uso de los bienes públicos.

De acuerdo con un modelo de la Organización Mundial de la Salud (OMS)(3), la salud y el bienestar están determinados por la interrelación de factores de diferente naturaleza, entre ellos, el desarrollo social y económico, la sanidad ambiental y disponibilidad de agua potable, la vivienda, la recreación, la posibilidad de tener trabajo y acceder a la educación, la prestación de los servicios de salud, la existencia de redes sociales, el tamaño de la familia y, finalmente, las características biológicas y el estilo de vida de las personas. A este modelo le agregamos otros determinantes que surgen, algunos de ellos nuevos, como la epidemia de sida, otros agentes reemergentes con dinámicas propias, como lo son la creciente violencia, la inseguridad social y la adicción y tráfico de narcóticos. Estos últimos representan desafíos para la salud pública y para la sociedad por sus efectos negativos sobre las comunidades.

Se reconoce hoy que el comportamiento agresivo o la conducta antisocial no se dan por generación espontánea sino que son aprendidos de las demás personas, del ambiente en que se vive, de lo que básicamente en la infancia y luego en la adolescencia y juventud se observa, se imita, se evita, se rechaza, se asimila o se combate (4-6). Por ello, hay que acudir a las ciencias sociales, a la epidemiología y a otras disciplinas científicas 
para estudiar las causas de la violencia social y acercarse así con certeza a entender el problema $y$, en consecuencia, proponer soluciones que se correspondan con tales hallazgos.

En ese propósito están comprometidas la OPS y la OMS, las que han definido que el papel del sector salud en lo que a violencia y lesiones se refiere, no se limita a mejorar la atención y rehabilitación de los heridos y víctimas que llegan a los hospitales, sino a evitar que las situaciones que preceden a su ocurrencia se presenten de nuevo. En el lenguaje de la salud pública se llama a esto con dos términos complementarios: promoción y prevención. La promoción de conductas y hábitos de vida saludables y la prevención de eventos dañinos para las comunidades y el individuo priman en el quehacer de la salud pública. La intención de las acciones preventivas de salud es evitar que los hechos ocurran o se agraven sus consecuencias. Para lograrlo, hay que basarse en el estudio de sus factores y causas y en la aplicación de medidas o intervenciones que muestren eficacia preventiva.

Los avances que se han logrado en salud pública para el control y prevención de enfermedades y promoción de la salud, se aplican igualmente al problema de la violencia. En consecuencia y en la búsqueda de alternativas preventivas, se propone abordar el problema con bases científicas, método válido y probado para la solución de otros problemas de salud. En síntesis: 1) caracterizar el problema en sus variables básicas de persona, lugar, tiempo, circunstancias y situaciones conexas; 2) identificar causas, asociaciones o factores de riesgo; 3) proponer intervenciones y evaluarlas, y 4) extender las intervenciones a otros sectores y difundirlas (7). No sólo el sector salud clama por la prevención (8), voces y propuestas desde otras disciplinas y ante conflictos colectivos igualmente sustentan la necesidad de buscar alternativas preventivas, concertadas, de largo plazo y sostenidas, muchas veces costosas pero que comparadas con el sufrimiento humano que la violencia trae consigo, su costo final es minúsculo (9).

Así, se requiere disponer de una definición operativa, consensuada, del objeto de estudio e intervención. De acuerdo con el Informe mundial sobre la violencia y la salud, de la OMS: "Violencia es el uso intencional de la fuerza física o el poder, real o por amenaza, contra la persona misma, contra otra persona, o contra un grupo o comunidad que puede resultar en o tiene alta probabilidad de resultar en muerte, lesión, daño psicológico, problemas de desarrollo o deprivación" (10). Se destacan en esta definición dos aspectos: la intencionalidad del acto violento y el daño que produce.

La violencia es, pues, por definición, un acto intencional que puede haber sido premeditado o planificado, como ocurre en los casos de asesinatos de líderes o enemigos, o las acciones del crimen organizado, o en los conflictos colectivos y las guerras. Otras veces, aunque sin planificación evidente, se ejecutan actos violentos con la intención de dominar, subyugar, intimidar o sencillamente demostrar supremacía o satisfacer un impulso a sabiendas del daño que se hace, así ocurre con la violencia contra mujeres, niñas y niños, ancianos o por las acciones de las pandillas juveniles. La intencionalidad distingue los actos violentos de aquéllos en los que también hay trauma físico o psicológico, como es el caso de la mayoría de las lesiones producidas en colisiones de tránsito, por ejemplo, pero que no se tuvo la intención de producirlas.

Por su parte, las Naciones Unidas adoptó en la Declaración sobre los Principios Básicos de Justicia para las Víctimas del Crimen y Abuso del Poder, la siguiente definición de víctima: "es toda persona que individual o colectivamente ha sufrido daño físico, mental, emocional, pérdida económica o sustancial desmejora de sus derechos fundamentales, a través de actos $u$ omisiones que son violatorios de las leyes que operan en ese Estado" (11) con la cual se complementa lo señalado antes sobre la intencionalidad del evento violento.

\section{Multicausalidad de la violencia}

Investigadores y académicos coinciden en que la violencia es un fenómeno multicausal, un solo factor o causa no es suficiente para explicar la complejidad ni las razones que impulsan o favorecen la ocurrencia de eventos violentos. La 
génesis de la magnitud y las variadas formas de expresión de la violencia social no se explican por razones genéticas. No es posible que incrementos de hasta cinco veces en las tasas de homicidios y del crimen, en períodos tan cortos como diez o veinte años, puedan explicarse por razones genéticas o características biológicas. En el modelo multicausal confluyen diversos niveles que se interconectan mostrando las relaciones entre ellos. Se comentan los que han sido mayormente estudiados o han mostrado mayor evidencia.

En el plano estructural macro a nivel de sociedad, se distinguen los factores relacionados con la desigualdad social, la pobreza, el nivel de desarrollo, la falta de oportunidades para la educación y el trabajo, el resquebrajamiento de valores como el respeto al derecho de los demás, la solidaridad, la corrupción, la rápida urbanización sin planificación y los acelerados cambios demográficos. Conviene señalar que la desigualdad social, medida por el coeficiente Gini, se ha asociado con el crimen y la violencia de tal forma que "un incremento de un punto en el coeficiente se correlaciona con un $1,5 \%$ de incremento en las tasas de homicidios y un incremento de 2,6\% en las tasas de robos, pero si la situación de desigualdad empeora permanentemente los efectos permanentes son 3,7 y 4,3 veces más altos para homicidios y robos"(12).

En un segundo plano, señalo aquellos factores institucionales adversos que llevan a la ciudadanía a perder su credibilidad tanto en las instituciones responsables o reguladoras de la convivencia ciudadana como en las que deben crear los ambientes propicios para la adecuada formación de valores y principios éticos. Por ejemplo, la poca eficacia y la corrupción de la policía y el sistema de justicia, en términos generales, generan un alto nivel de impunidad y conducen a la pérdida de la confianza de los ciudadanos en su capacidad de protegerles y ser refugio a la inseguridad y la violencia. En buena hora, en muchos países se ha avanzado o se han iniciado procesos de reforma de la policía y del sistema de justicia que cambiarían la visión de la comunidad sobre las mismas. Por su lado, la mala calidad de la educación actúa como un factor que impide a muchas personas el acceso a fuentes de trabajo y a avanzar en su proceso de formación de ciudadanía.

La familia, entendida en este contexto como la institución básica de la sociedad, y en ella, el padre y la madre, juega un papel trascendental para la educación y formación de la personalidad, para la identificación de la persona como sujeto social. Es en la familia donde se inicia ese proceso para entender y asimilar principios de respeto y acatamiento de las normas de convivencia. En el hogar, las primeras experiencias de niñas y niños marcarán en gran medida su comportamiento como adultos. Si el hogar es escenario de violencia intrafamiliar y conyugal, la probabilidad de adoptar conductas similares en el futuro se incrementa (13), o si la educación es muy permisiva o sobreprotectora, sin normas, las probabilidades de adoptar conductas violentas 0 criminales se incrementa; así se observó en un estudio de la historia de vida de 50 hombres homicidas entrevistados en la cárcel comparadas con las de otros 50 sin historia de criminalidad, agrupados por edad y barrio de procedencia y entrevistados en sus hogares (14).

Finalmente, en un tercer plano, se distinguen los factores directos, facilitadores o circunstanciales los cuales, en situaciones específicas, son desencadenantes o facilitan la ocurrencia de eventos violentos. El porte de armas, el consumo excesivo de alcohol, el uso de narcóticos, la falta de espacios de recreación y la falta de iluminación en espacios públicos, por ejemplo, están claramente correlacionados con la ocurrencia de hechos de violencia. Un estudio sobre los efectos de la prohibición del porte de armas en Cali y Bogotá, atribuye a esta medida una disminución de $13 \%$ en la tasa de homicidios (15).

Los tres planos mencionados están interconectados y cuando ocurren en conjunto, se sinergizan e incrementan los riesgos. La visión multicausal de la violencia implica que su estudio y abordaje preventivo sea multisectorial e interdisciplinario, con el concurso activo de las comunidades. 


\section{Momentos históricos de intervención de violencia}

Considero que el abordaje gubernamental y social de la violencia ha tenido una evolución que ha pasado por tres etapas:

1) Represión y control: a cargo de la policía y el sistema judicial. Las teorías basan esta acción en el efecto intimidatorio que sobre los potenciales agresores tuviese la reclusión carcelaria y pérdida de derechos con que el estado castiga a quienes trasgreden la ley, política que no ha mostrado tener la efectividad pregonada (16). Este enfoque ha sido el tradicional y aunque aislado no produce los efectos esperados, tampoco se puede olvidar que quien comete un crimen o ejerce violencia debe someterse a la ley, la cual si es justa, equitativa y respeta los derechos humanos, debe ser inequívocamente aplicada, hoy y siempre.

2) Prevención: la necesidad de abordar la violencia desde una perspectiva preventiva amplia, ha tenido un eco creciente en las últimas tres décadas. El enfoque de salud pública ha sido un aporte esencial en la adopción de políticas de prevención (17). Es la respuesta intersectorial a la multicausalidad de la violencia. Hoy es reconocido y aceptado que los diversos sectores que tienen que ver con la atención, rehabilitación, cuidado y control a víctimas y victimarios de actos violentos, deben unir esfuerzos en la prevención. En esta perspectiva, la acción intersectorial busca responder a la multicausalidad de la violencia, actuando de manera preventiva e integradora de tal forma que a la par que enfrenta al hecho delictivo busca construir ciudadanía (18).

3) Recuperación del tejido social y humano: la violencia produce y genera daños en la estructura social y fomenta comportamientos que horadan las bases y los principios de convivencia y resolución de conflictos. La ética social, por la cual se da el respeto al derecho ajeno, la convivencia y la solidaridad entre conocidos, vecinos y comunidad en general, o lo que el filósofo y ensayista Savater define como la "búsqueda por mejorar a las personas"(19) en su relación con los demás, se encuentra seriamente afectada, en particular en países donde los niveles de violencia son altos y sus expresiones son múltiples y cotidianas. La violencia tiene raíces históricas y su solución pasa por la restauración de la funcionalidad social, de sus instituciones básicas formales y no formales, desde la familia.

El proceso de la violencia no acaba con una víctima, es sólo su expresión de momento. Las instituciones deben atender los diversos aspectos que explican la violencia y la ruptura de los lazos de convivencia que hacen a una sociedad más vulnerable a la violencia que otras. El capital social, también llamado tejido social por su implicación a construir redes sociales, va de la mano con nuestra forma de relacionarnos con las demás personas, respetar sus derechos y fomentar la solidaridad. Esta perspectiva, que ha cobrado fuerza desde hace unos diez años, demanda intervenir en la prevención de la violencia, bajo un compromiso muy amplio de los ciudadanos, líderes, jerarcas, comunicadores, educadores y, en fin, la sociedad toda para recuperar la ética, su capital humano y social.(20)

Hoy en día, los tres momentos descritos deben coincidir para incidir de manera positiva y holística en la solución del problema. No son excluyentes. A mayor relación entre ellos mejores las posibilidades de éxito. Gobiernos, ONG y comunidades deben unir y mantener sus esfuerzos por este propósito común. Esta concepción se identifica plenamente con el recientemente publicado concepto de salud pública, por la OPS: "La salud pública es el esfuerzo organizado de la sociedad, principalmente a través de sus instituciones de carácter público, para mejorar, promover, proteger y restaurar la salud de las poblaciones por medio de actuaciones de alcance colectivo".(21)

\section{Magnitud en el continente americano}

Mortalidad por homicidios y suicidios: aun cuando no todos los países disponen de cifras confiables sobre la ocurrencia de las distintas formas de violencia, las cifras disponibles son suficientes para mostrar lo alarmante de la situación. En síntesis y según registros oficiales recibidos en OPS (22), cerca de 115.000 personas mueren asesinadas cada año en las Américas; la mayoría de ellos son hombres, en algunos países más del $85 \%$ de los casos; 55.000 cometen 
suicidio, con una tendencia hacia edades más jóvenes. En Suramérica, Brasil tiene las tasas más altas de homicidio y Chile la más baja entre los países del cono sur (figura 1), pero en la parte norte Colombia supera no solo a Brasil sino a todos los países de la región (figura 2). En Norteamérica, México tiene la tasa más alta y Estados Unidos (23) , aunque con notorio descenso en los últimos 10 años, ostenta la más alta de los países desarrollados del mundo (figura 3 ).

Violencia intrafamiliar: por encuestas diversas se ha determinado que en 20 a $60 \%$ de los hogares se ejerce alguna forma de violencia intrafamiliar. Si este porcentaje se convierte en cifras se determina que millones de niños, niñas y mujeres sufren violencia por parte de personas cercanas (24). Una amplia red de instituciones y ONG han logrado la creación de leyes de protección a las mujeres contra la violencia intrafamiliar y conyugal y han avanzado en la visualización del problema y han logrado la atención de un número creciente de organizaciones e instituciones que se unen al coro de la no violencia, pero aun cuando hay avances en este sentido aún falta un gran camino por recorrer, millones de mujeres y niños sufren la violencia intrafamiliar (25).

La Ruta crítica de la mujer que sufre violencia es una investigación realizada en varios países que ha arrojado luces sobre lo que se debe hacer para prevenir nuevos o repetidos casos (26-32). Los factores que se relacionan con esta situación se han estudiado en diferentes contextos y, si bien son específicos del país o ciudad donde se realizan, también se ha mostrado consistencia de hallazgos de país a país. Así, por ejemplo, en Nicaragua, se encontró que la violencia era más prevalente entre mujeres con los siguientes factores de riesgo: más de 5 hijos; con historia de violencia en sus hogares de origen (el de su propia madre y el de su suegra); sin trabajo fuera del hogar y en niveles de pobreza; jóvenes y con poca o ninguna educación, y viviendo en zonas urbanas (33).

Violencia juvenil: me detengo un poco en lo de violencia juvenil porque considero que es un problema social que no se ha atendido adecuadamente. El alarmante aumento de

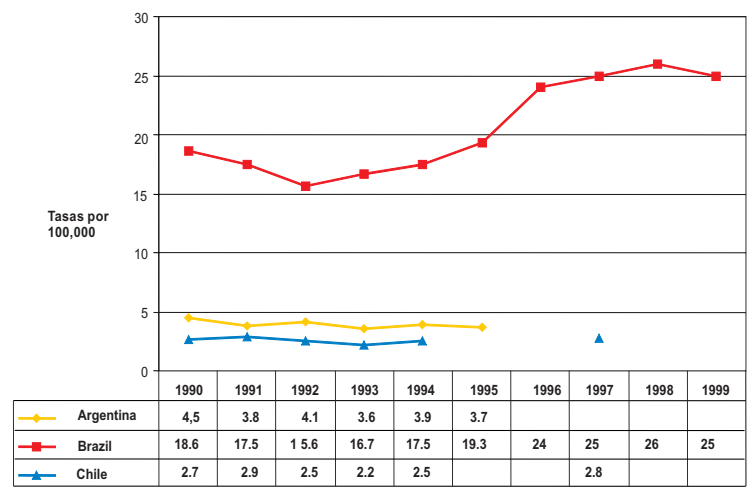

Figura 1. Tasas de homicidios en el Cono Sur de Suramérica,1990-1999.

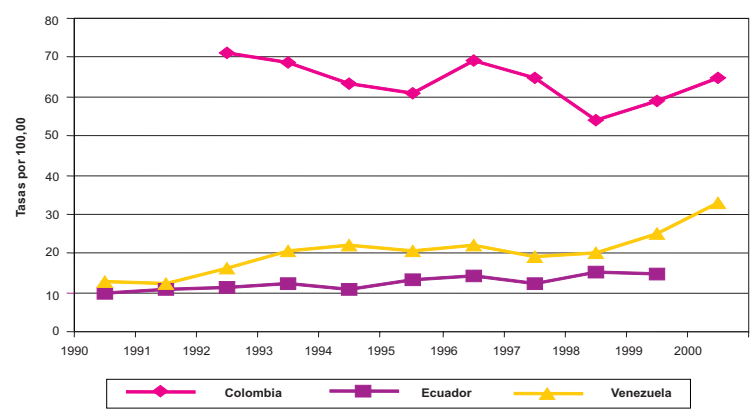

Figura 2. Tasa de homicidios en países andinos de Suramérica.

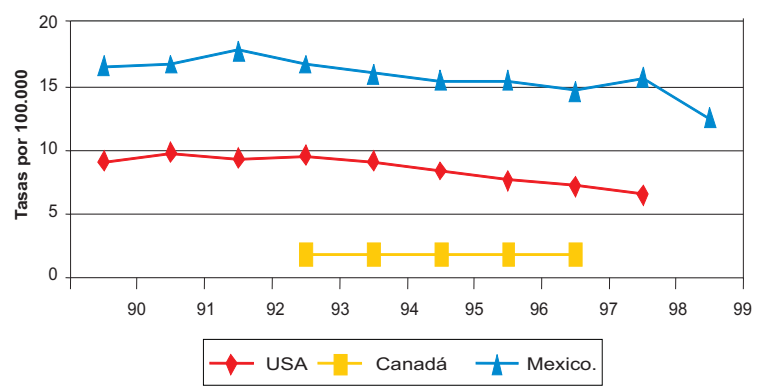

Figura 3. Tasas de homicidio en Norteamérica. 1990-1999.

pandillas juveniles involucradas en actividades violentas y criminales, en las que se agrupan por miles de jóvenes desde edades tan tempranas como los 8 o 10 años, es un fenómeno social al que no se ha respondido adecuadamente (34-35). La violencia es la primera causa de muerte entre jóvenes hombres de 15 a 29 años en varios países, con un incremento significativo de los años perdidos de vida $(36,37)$. En Honduras y El 
Salvador, sólo para citar dos casos preocupantes pero no los únicos, se estima que en cada uno de estos países hay 30.000 o más muchachos y muchachas en 'maras' o pandillas juveniles, los cuales consumen drogas, producen heridos y muertos en enfrentamientos con bandas rivales y la policía y, en ocasiones, entre la población general, además de violaciones, robos y atracos. Son también el caldo de cultivo del crimen organizado. Se han identificado grupos de este tipo en Jamaica $(38,39)$, Costa Rica, Perú, Colombia, México, Estados Unidos y aún en Chile (40).

La violencia juvenil se ejerce básicamente en pandillas. Es la que en forma repetida ejercen jóvenes entre 10 y 24 años, la mayoría de las veces como miembros de un grupo en el que puede o no haber jerarquía organizativa. Su aparición no es súbita. Entre los factores identificados se destacan el económico-social, la falta de oportunidades educativas, laborales y de recreación, así como la ausencia de mecanismos de participación social. En el ambiente familiar, han vivido la violencia intrafamiliar como víctimas o testigos, bien sea del padre hacia la madre, o de uno de los progenitores hacia los hijos e hijas; o sufren las consecuencias de la desintegración de su propia familia, en la que el padre está ausente y la madre lleva un papel sobredimensionado, al que no siempre puede responder, con consecuencias negativas en lo psicológico-afectivo y de identificación de papeles.

Los futuros pandilleros viven en ambientes cargados de violencia, asimilando la subcultura de la violencia como la adecuada para resolver conflictos, independientemente de la naturaleza de los mismos. El espacio fuera del hogar se convierte en un 'refugio' alterno al familiar y en ese ambiente social viven emociones inmediatas que su espíritu juvenil les reclama. Allí viven intensamente, se identifican con sus pares, crean círculos sociales y aprenden sus propias formas de relacionarse con la sociedad. El acceso a narcóticos, alcohol, cigarrillo y armas cortantes o de fuego, a tan temprana edad como 7 o 10 o 15 años, facilita la distorsión de la 'otra' realidad, la que se vive por fuera de la pandilla (figura 4).
A partir de experiencias, conversaciones y estudios sobre el tema, se ha construido el siguiente modelo que analiza la ruta que siguen muchos jóvenes involucrados en pandillas(41) (figura 5). El modelo se sustenta en dos pirámides con vértices en direcciones opuestas. En la pirámide con base amplia se clasifican los grupos humanos según su relación con los jóvenes. La pirámide con vértice hacia abajo agrupa las actividades que en forma progresiva van cometiendo quienes siguen una ruta delictiva. Hay una relación inversa entre la amplitud de la base social en que se vive o participa y las actividades

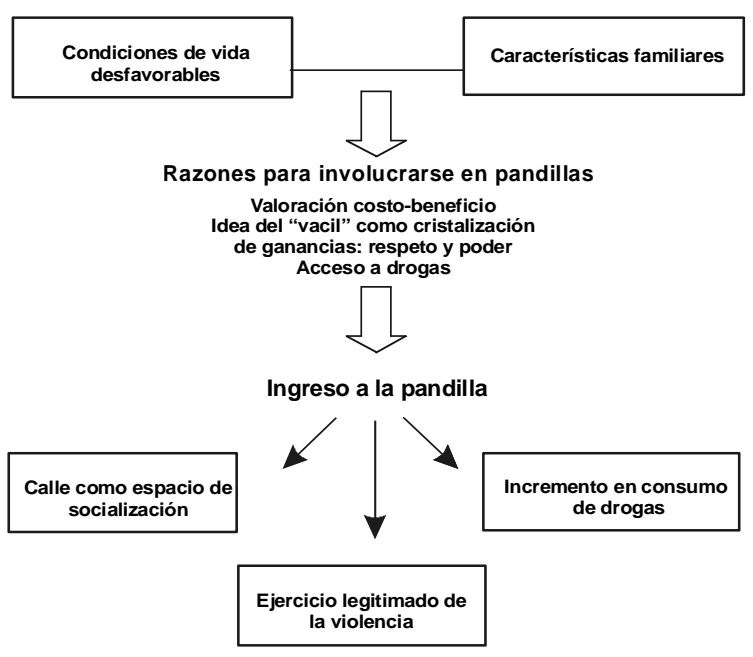

Figura 4. Modelo 3 (34).

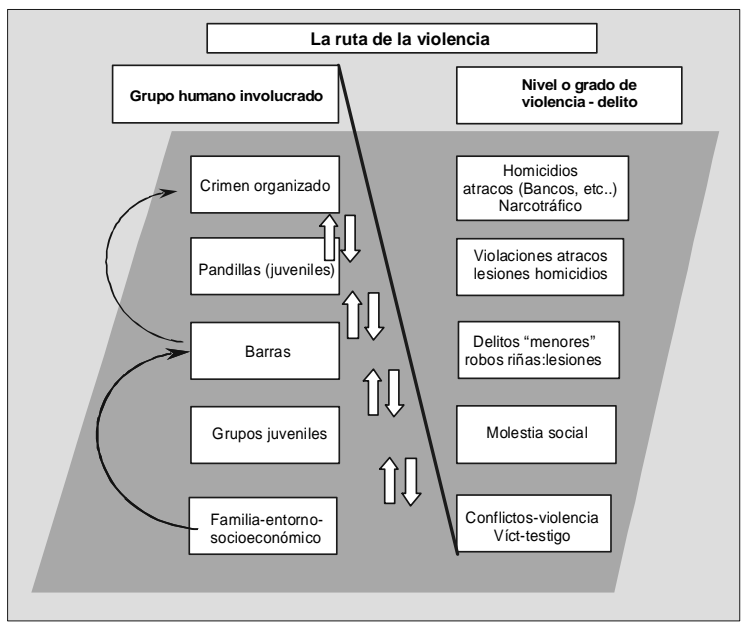

Figura 5. Modelo 4. 
de carácter antisocial o criminal que se cometen. Entre mayor es la base social, menor la gravedad del hecho delictivo. Las flechas indican que la ruta no es lineal sino que la pertenencia a los grupos es dinámica, los jóvenes 'entran y salen' de ellos por diversas razones, las cuales que hay que considerar para el diseño de proyectos preventivos.

En la base de la pirámide social están el entorno socioeconómico y la familia, el hogar, donde los niños y las niñas pueden ser testigos o víctimas de violencia, u observan cómo los adultos resuelven sus diferencias acudiendo al uso de la violencia, la fuerza o la intimidación. Las familias de origen son la base 'institucional' donde se inicia ese camino que algunos seguirán y otros abandonarán por diversas razones. Esto trae efectos negativos de corto o largo plazo para su formación. Tal potencial efecto negativo no se da sólo por el hecho de recibir golpes u observar que la madre es golpeada, o ver cómo los vecinos y la sociedad confrontan sus realidades (caso de países en guerra o en conflictos sociales de alguna magnitud), sino que allí en esos medios de aprendizaje que son la casa, el barrio, la ciudad, los niños y niñas reciben enseñanzas de cómo se dirimen las disputas: a golpes, con violencia, usando armas.

Hoy se sabe que, afortunadamente, aunque muchos viven experiencias agresivas o son testigos de violencia en su infancia o adolescencia, no llegarán a ser adultos agresores. Por el contrario, desean y luchan por no repetir los errores y conductas aprendidos en el hogar o en sus comunidades. Estos son los que recibieron cariño, amor, ternura, influencias positivas no siempre identificadas $y$, en fin, tuvieron la posibilidad de superar la adversidad y los efectos negativos de la agresividad, estuvieron expuestos de una u otra forma (la cual no siempre se logra conocer) a factores de 'protección' de su desarrollo emocional y personal. A esto se llama hoy 'resilience' ('elasticidad'). Su conducta futura no será violenta.

En un segundo momento, los jóvenes en forma por demás necesaria se unen a grupos afines por edad o intereses, donde sus prácticas sociales pueden ser llamadas 'molestias sociales', no son actividades delictivas ni criminales, pero sí perturban la convivencia tal como escuchar música a alto volumen, jugar en la calle hasta altas horas de la noche o, simplemente 'vacilar' en grupo y crear temores entre los vecinos. En este momento, los riesgos de seguir en la ruta de la violencia son otros, ya no ajenos a la cotidianidad del joven sino inmersos en su propio desarrollo. Hay que señalar que la agrupación juvenil per se no sólo no debe ser rechazada sino que es saludable, estimulable. La socialización pasa por la identificación con las personas con intereses y realidades comunes, las cuales se comparten con pares de la misma edad y condición social.

El riesgo de continuar en esta escala se da cuando las oportunidades de acceder a otras formas de socialización son más débiles que las de continuar experimentando en la calle los desafíos que el grupo empieza a enfrentar. Nunca en esta ruta el joven es un ser aislado, por el contrario, es sujeto de las influencias sociales, emocionales y económicas que va encontrando en su camino. Las experiencias, carencias, negaciones o realizaciones de vida contribuyen a moldear el futuro de las personas. Los jóvenes y sus grupos los van construyendo. En las agrupaciones juveniles hay de hecho un potencial humano inmenso al cual hay que entender, exigir y responder con claridad.

Un peldaño más cercano a la delincuencia y la criminalidad se observa en las llamadas 'barras' o conjunto de jóvenes que en número reducido se involucran en delitos menores como robos, hurtos o riñas con barras rivales en las que son heridos. El consumo de drogas legales e ilegales se hace más común. La deserción escolar tiende a aumentar. Es un momento crucial y complejo, puesto que si se permanece en estas actividades y se avanza en la criminalidad, o se abandona este tipo de vida, los jóvenes están definiendo su futuro.

El escalón más cercano a la delincuencia organizada es el de las pandillas juveniles. En éstas hay intereses económicos, no siempre hay jerarquía, pero suele darse, mayor realización de actividades delictivas y violentas, asesinatos, 
violaciones sexuales, atracos, robos enfrentamientos con miembros de la policía, uso de distintivos como los tatuajes o simbolismos que los unen e identifican. Las muchachas pandilleras son mal tratadas, abusadas sexualmente y su papel no es el mismo que el de los muchachos. De alguna manera, reproducen actitudes y subvaloraciones que algunos sectores sociales tienen de la mujer. Sin embargo, mantienen, valoran y protegen a sus madres. En este nivel, los lazos de pertenencia al grupo son fuertes pero no irrompibles.

Las pandillas se convierten en caldo de cultivo del crimen organizado, de los narcotraficantes, de los asaltantes de bancos o joyerías, de las bandas armadas al servicio de intereses oscuros. Estos grupos desarrollan organizaciones complejas, con mayores recursos, criterio empresarial, tecnología avanzada y contactos e infiltraciones en el sistema social y político. La propia organización del delito requiere de nuevos insumos: el sicario, el pandillero, el gamín, todos de condición juvenil. En algunos países son entrenados y se les paga por matar. El punto de retorno a la no violencia es cada vez más difícil.

No se trata sólo de analizar este proceso sino encontrar dentro de esa escala dónde y cómo actuar para evitar que más y más muchachos y muchachas accedan a acciones de mayor riesgo en la ruta del crimen y la violencia. A más temprana edad cronológica y de intervención, mayores las posibilidades de acertar. Los jóvenes y la sociedad en general reclaman políticas estables y sostenidas para enfrentar la demanda de necesidades a la que ellos tienen derecho. En la medida en que las necesidades sociales de la juventud se resuelvan, también se estará enfrentando de una manera positiva el problema social que significan las pandillas juveniles.

Otras formas de violencia y crimen que tienen relación con la violencia social e interpersonal son las guerras y los conflictos internos (Colombia es el único país de las Américas con este tipo de conflicto en la actualidad) o internacionales, la violencia política de grupos o contra dirigentes, los secuestros extorsivos y políticos, los linchamientos, la violencia multinacional por el crimen organizado y las mafias con su tráfico de narcóticos, el comercio sexual y el contrabando de armas que dificulta el control de la distribución de las mismas.

\section{Impactos sociales y económicos}

El incremento de los eventos violentos, la aparición de formas modernas y tecnificadas de hacer violencia y el cambio en la percepción de la población provocan efectos sociales y para el desarrollo, tales como:

1) En lo social, la calidad de vida reduce la posibilidad de disfrutar los espacios públicos y el tiempo para disfrutarlos. Hay temor, fundado o no, para el desplazamiento a lugares y sitios especiales. Si se dispone de recursos, se cierra el espacio privado, con rejas y guardas de seguridad (42).

2) La demanda de gastos por violencia representa una proporción significativa del PIB. Un estudio del Banco Interamericano de Desarrollo (BID) calculó en cerca de $14,2 \%$ del PIB en América Latina, o sea, US $\$ 168.000$ millones, la inversión hecha para atender los costos directos, indirectos y por transferencias de la violencia (43). A esta conclusión se llegó después de determinar con métodos epidemiológicos y econométricos los costos de la violencia en seis países de América Latina (cuadro 1), entre ellos, Colombia con el impacto más negativo.

Otro estudio muestra los efectos que los indicadores macroeconómicos tienen sobre los índices de crimen y violencia; así, por el lado positivo, un aumento de $1 \%$ del PIB produce una disminución de la tasa de homicidios de 2,4\% y en la tasa de robos en 13,7\%; mientras que, por la vía negativa, un aumento de $1 \%$ en el coeficiente Gini lleva a un aumento en la tasa de homicidios de $1,5 \%$ y en la de robos de $2,6 \%$ (12).

El efecto que produce la violencia en el desarrollo económico de un país es significativo porque impone restricciones a la economía de una nación; tanto por las pérdidas como por el desestímulo a la inversión por la falta de estabilidad y temor a los secuestros y asesinatos; el ahorro interior se reduce y quienes pueden colocan sus capitales 
Cuadro 1. Costos de la violencia social en países en porcentaje del PIB, 1997-BID 2000.

\begin{tabular}{lcccccc}
\hline Costos & El Salvador & Colombia & Venezuela & Brasil & Perú & México \\
\hline Directos & 9,2 & 11,4 & 6,9 & 3,3 & 2,9 & 4,9 \\
Indirectos & 11,7 & 8,9 & 4,6 & 5,6 & 1,6 & 4,6 \\
Transferencias & 4,0 & 4,4 & 0,3 & 1,6 & 0,6 & 2,8 \\
Total & 24,9 & 24,7 & 11,8 & 10,5 & 5,1 & 12,3 \\
\hline
\end{tabular}

en el exterior; ambas situaciones disminuyen prospectos del futuro económico.

3) Para el sector salud, los efectos se ven, en primer lugar, en la enorme inversión de recursos de salud hacia lesiones y emergencias provenientes de la violencia. El estudio realizado por el BID en seis países de América Latina calculó en US $\$ 2.000$ millones los costos de atención médica y en US $\$ 17.000$ millones la destrucción del capital humano por muerte prematura o discapacidad entre víctimas de hechos violentos. Por países, los costos en salud fueron, en porcentajes del PIB, de 4,3 en EI Salvador; 5,0 en Colombia; 0,3 en Venezuela; 1,9 en Brasil; 1,5 en Perú, y 1,3 en México (43).

A nivel intrafamiliar hay un impacto negativo en el rendimiento de los niños en edad escolar cuando son testigos y víctimas de la violencia en sus hogares. Las mujeres víctimas de violencia suelen ser menos productivas en el trabajo y tienden a ser despedidas con más frecuencia. En Chile y Nicaragua, se estimó que los ingresos de las mujeres violentadas es inferior en 57 y $39 \%$, respectivamente, con respecto a las que no sufren violencia intrafamiliar; son ellas las que tienen mayor ausentismo laboral (44).

Entre los costos directos se contabilizan los del sistema de salud, la policía, el sistema de justicia criminal, los de vivienda y transportes a los juzgados y servicios de salud, y los de los servicios sociales.

Entre los costos no monetarios, se detecta mayor morbilidad, mayor mortalidad debido a homicidios y suicidios, abuso de alcohol y drogas, desórdenes depresivos y una amplia gama de desórdenes psicosomáticos o somáticos reflejados en el aparato digestivo y reproductivo.

\section{Prevención de la violencia}

La salud pública es una práctica social de naturaleza interdisciplinaria de responsabilidad del Estado de tal manera que asegure el acceso y la calidad de la atención de salud y la prevención del daño. Para ello se requiere diseñar estrategias, trabajar intersectorialmente, apoyar proyectos y definir planes nacionales o municipales que respondan a la situación particular de cada país o ciudad.

En el caso de la violencia, las acciones de prevención primaria buscan fomentar un ambiente social e individual de respeto y tolerancia por la diferencia, de valores sociales y de conducta personal que favorezcan que los conflictos se resuelvan de maneras no violentas, o sea, se dirigen a evitar que ocurra el hecho violento. Las estrategias destinadas a disminuir la pobreza y la brecha entre ricos y pobres, a buscar la equidad social y de género, a mejorar la educación, a la recuperación de la ética y el control social, a capacitar a las personas para la solución pacífica de los conflictos, a mejorar la educación y diálogo de padres y madres con sus hijos/as son algunos ejemplos de este nivel de prevención.

La prevención secundaria se aplica cuando un evento violento ya ha ocurrido y su intención es evitar nuevos episodios, o que sean de mayor gravedad. Es necesario en todo caso que los profesionales estén capacitados y motivados para detectar casos y apoyen los programas de tamizaje y vigilancia epidemiológica, y no limitarse solamente a la atención de las lesiones. Ejemplos de este tipo de prevención secundaria lo constituyen los programas donde se identifican las personas que van a las instituciones de salud por haber sufrido una herida violenta y son vinculadas activamente a programas de 
intervención en crisis, a través de los cuales se buscan soluciones a las situaciones que precedieron el evento violento (45 y 46). Las consejerías para la mujer y los niños, donde se les ofrece respaldo y atención para la solución de violencia intrafamiliar, las visitas de hogar para víctimas de violencia por personal entrenado en consejería (47-50). Las redes y grupos locales que trabajan por la prevención de la violencia, además de la capacitación dirigida al personal de salud, son hoy alternativas de prevención en varios países $(51,52)$. También se dirigen a los agresores, tanto hombres como mujeres. Los grupos de masculinidad y las Escuelas de Padres buscan modificar conductas agresivas en las relaciones intrafamiliares y de pareja. Otro caso es el de programas para jóvenes miembros de pandillas juveniles donde, con su participación, se analizan y buscan opciones de vida diferentes, como el Programa Polígono Industrial y Homies Unidos en San Salvador (53) .

La prevención terciaria se aplica en salud pública para evitar mayores daños a quienes tienen ya una enfermedad establecida, a mejorar su calidad de vida, es la rehabilitación. En el caso de la violencia se aplica, por ejemplo, a quienes hayan sido condenados y se encuentren cumpliendo penas de prisión. Los programas con tal fin deben enfocarse a la enseñanza de actividades productivas, acompañamiento psicológico y de resocialización. La prevención terciaria es de especial importancia en el caso de jóvenes recluidos por infracciones menores y que por ausencia de rehabilitación, con mucha frecuencia, el período de confinamiento se vuelve más bien de perfeccionamiento de la actividad delictiva.

La prevención de la violencia tiene una doble dimensión: la objetiva, referida a los hechos concretos de violencia y, la subjetiva, que tiene que ver con la percepción y la representación que el ciudadano se hace de ella. El Estudio multicéntrico sobre actitudes y normas culturales frente a la violencia, ACTIVA, (54) coordinado por la OPS, a través de encuestas a 10.821 adultos, permitió conocer cómo se percibe y se vive la violencia y la inseguridad en siete ciudades de América Latina y en Madrid, España. ACTIVA es una contribución de gran valor para el esclarecimiento del problema de las actitudes y normas hacia la violencia y, también, permitió establecer líneas de base para futuras investigaciones en el área de la victimización.

La violencia es prevenible y entre más pronto se empiece, tanto desde temprana edad en la vida como con diversos proyectos, se obtendrán resultados de mayor costo/beneficio; sin embargo, es la sostenibilidad de los programas y proyectos lo que permite garantizar su impacto positivo, su capacidad para lograr los cambios propuestos. El fin es reducir la ocurrencia de violencia, sus causas, consecuencias y factores de riesgo, a través de la promoción de los factores protectores y el control de los factores de riesgo.

\section{La necesidad de sostener los programas}

Con el propósito de mostrar la validez del enfoque preventivo y la necesidad de impulsarlo en forma decidida y sostenida, menciono los casos de Bogotá y Cali, que ilustran el impacto cuando los programas se mantienen integrales y se continúan en el tiempo (figura 6).

Bogotá. En Bogotá, desde 1995, se inició un programa de convivencia y seguridad ciudadana basado en un enfoque desde la cultura y la investigación. Se definió la necesidad de estudiar porque ocurren hechos de violencia y proponer soluciones al problema. Distintas administraciones desde ese año continuaron aplicando políticas de prevención de violencia y promoción de seguridad, la mayoría de los proyectos con financiación local.

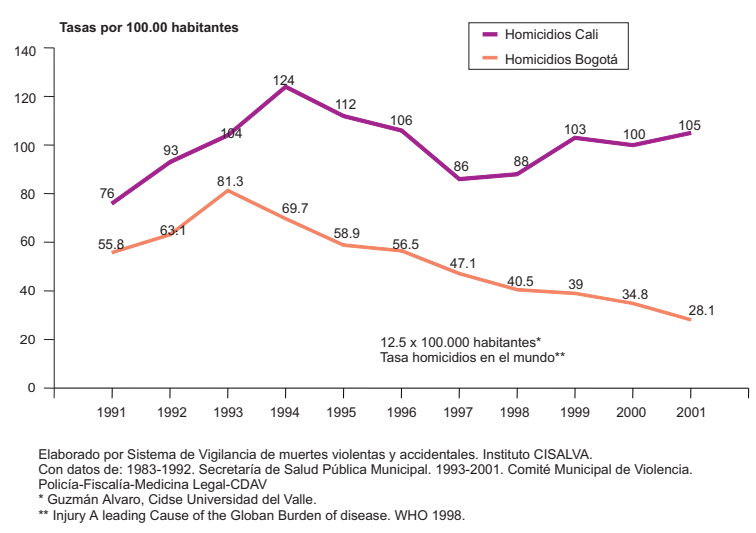

Figura 6. Tasas de homicidios en Cali y Bogotá, 1991-2001. 
La teoría de este programa se basa en reconocer un "divorcio entre tres sistemas reguladores del comportamiento: ley, moral y cultura". Tal divorcio se expresa en una "carencia de aprobación moral o cultural de las obligaciones legales y aprobación cultural o moral de las acciones ilegales"(55). Para resolver el conflicto social que crea la ruptura, su propuesta se centra en la reeducación, de adultos en particular, a través del programa "Cultura ciudadana" traducida en acciones de gobierno como el cierre más temprano de establecimientos donde se vende licor, el uso de mimos callejeros para mejorar el caos vehicular, programas diversos de acercamiento a la ciudadanía, desarme y otros cuya continuidad y constancia han logrado disminuir la tasa de homicidios por 100.000 hab de 80 en 1993 a 30 en 2001, es decir, una disminución de más del $62,5 \%$ en siete años. Otros delitos también han disminuido (56).

Cali, Colombia. En 1992, la alcaldía lanzó el programa "Desarrollo, seguridad y paz", conocido como DESEPAZ (57), también con una serie de estrategias polivalentes y amplia participación ciudadana. Las muertes por homicidios por 100.000 habitantes disminuyeron de su pico más alto en 1994, de 124 a 86 en 1997, o sea, una disminución del $30 \%$ en 3 años. Igualmente disminuyó la mortalidad por accidentes de tránsito y otras formas de violencia. Lamentablemente el programa DESEPAZ, en su integralidad preventiva, perdió apoyo en las administraciones siguientes y la situación ha vuelto a agravarse, al punto que la tasa de homicidios ascendió a 88, 103, 100 y 105 por 100.000 hab desde 1998 a 2001.

\section{Comentario final}

La disminución de hechos violentos será exitosa si se incorporan diversas estrategias y líneas de acción diseñadas, financiadas y sostenidas de común acuerdo con las autoridades y comunidades, bajo criterios de respeto y participación de la sociedad en general. La OPS y la OMS refrendan su compromiso para apoyar la búsqueda de soluciones a este problema social y de salud.

Los siguientes criterios forman la base de las políticas de la OPS para la prevención de la violencia: 1) obedece a causas múltiples y debe ser enfrentada con estrategias polivalentes; 2) es necesario mejorar y mantener actualizada la información sobre las características y circunstancias en que ocurren los hechos de violencia; 3 ) se debe privilegiar lo preventivo sobre lo represivo, con énfasis hacia los proyectos que actúen sobre las causas que pueden evitar la ocurrencia de violencia (prevención primaria), sin descuidar la atención y rehabilitación de las víctimas; 4) las intervenciones, proyectos 0 decisiones tomadas por autoridades, instituciones académicas y ONG deben hacerse con la debida planeación, seguimiento y evaluación de sus resultados; 5) las comunidades deben ser parte activa de los proyectos, con claro derecho a proponer, modificar y actuar en el desarrollo de los mismos; 6) la sociedad civil (entendida como los sectores sociales que no forman parte del aparato del estado, gobierno, fuerzas militares, legisladores, jueces y magistrados) tienen responsabilidad y derecho a apoyar e implementar acciones y proyectos preventivos, y 7) las condiciones locales y las comunidades dan la pauta para el diseño de políticas.

Las nueve recomendaciones contenidas en el Informe mundial sobre la violencia y la salud (58), son de por sí herramientas de trabajo, ellas son: 1) crear, poner en marcha y supervisar un plan nacional de acción para prevenir la violencia; 2) mejorar la capacidad para recolectar datos sobre la violencia; 3) definir las prioridades y fomentar la investigación de las causas, consecuencias, costos y prevención de la violencia; 4) promover la prevención primaria; 5) reforzar las respuestas a las víctimas de la violencia; 6 ) integrar la prevención de la violencia en las políticas sociales y educativas, promoviendo al mismo tiempo la igualdad social y entre los sexos; 7) incrementar la colaboración y el intercambio de información sobre la prevención de la violencia; 8) promover y supervisar el cumplimiento de los tratados internacionales y la legislación y otros mecanismos de protección de los derechos humanos, y 9) buscar en el ámbito internacional respuestas prácticas y consensuadas al tráfico mundial de drogas y de armas.

La solución y el compromiso para cambiar la actual situación de violencia no son de corto plazo, 
pero debemos empezar más pronto que tarde con la decisión de sostener y financiar los proyectos que bien sean prometedores, tengan un buen diseño o hayan tenido éxito en otras latitudes. Con William Ospina, decimos "necesitamos con urgencia una filosofía del crimen, ya que el hombre es el único animal que mata sin necesidad" (59).

\section{Referencias}

1. Ordenes $\mathbf{O}$, Vanolli $\mathbf{H}$, editores. Sociedad, violencia y salud. Memorias, Conferencia Interamericana sobre Sociedad, Violencia y Salud, noviembre 1994. Washington, D.C.: OPS/OMS; 1995.

2. Alleyne G. La prevención de la violencia: compromiso para la organización. Rev Panam Salud Publica1999;5: 1.

3. Tarimo E, Webster EG. Primary health care concepts and challenges in a changing world. Alma-Ata revisited. WHO/SHS/CC/94.2. SHS paper number 7.

4. Bandura A. Aggression: a social learning analysis. Englewood Cliffs, NJ: Prentice Hall; 1973.

5. Bandura A. Social foundations of thought and action: a social cognitive theory. Englewood Cliffs, NJ: Prentice Hall; 1986.

6. McAlister A, Vélez LF. Behavioral sciences concepts in research on the prevention of violence. Pan Am J Public Health 1999;5:316- 21.

7. Hammond R. The public health model for violence prevention: the CDC perspective. En: Violence and health. Proceedings, WHO global symposium. Kobe, Japan, Oct. 1999. Kobe: WHO Kobe Centre for Health Development; 2000.

8. Prothrow-Stith D. The epidemic of youth violence in America: using public health prevention strategies to prevent violence. Journal of Health Care for the Poor and Undeserved 1995;6.

9. Carnegie Commission on Preventing Deadly Conflict. Preventing deadly conflict: final report. Washington, D.C.: Carnegie Commission on Preventing Deadly Conflict; 1997. p.7,8.

10. Krug EG et al., editors. World report on violence and health. Geneva: World Health Organization; 2002. p.5.

11. United Nations. On the use and application of the United Nations Declaration of Basic Principles of Justice for Victims of Crime and Abuse of Power. En: International Victim Assistance Handbook. New York: Unicef; 1998.

12. Fajnzylber $\mathbf{P}$, Lederman $D$, Loaiza $N$, editores. Crimen y violencia en América Latina. Bogotá, Colombia: Editorial Alfaomega; 2001.

13. Astbury $\mathbf{J}$ et al. The impact of domestic violence on individuals. Med J Aus 2000;173:427-31.
14. Banguero H, Castro E, Concha-Eastman A, de Roux G, Echeverri O, Escobar J, et al. Dimensionamiento y costos de la violencia en Colombia. Washington, D.C.: Banco Interamericano de Desarrollo; 1998.

15. Villaveces $\mathbf{A}$, et al. Effect of a ban on carrying firearms on homicide rates in 2 Colombian cities. JAMA 2000; 283:1205-9.

16. INREDH. Conferencia Regional sobre la situación carcelaria en la Región Andina. Quito, Ecuador: Ed. INREDH; 2000.

17. Krug EG, et al, editors. Violence, a global public health problem. En:World report on violence and health. Geneva: World Health Organization; 2002.

18. Reiss AJ, Roth J, editors. Understanding and preventing violence: panel on the understanding and control of violent behavior. Washington, D.C.: National Academy Press; 1993.

19. Savater F. Etica, política y ciudadanía. México: Editorial Grijalbo; 1998.

20. Moser C, et al. Violence in Colombia. Building sustainable peace and social capita. A World Bank country study. Washington, D.C.: The World Bank; 2000.

21. Organización Panamericana de la Salud. La salud pública en las Américas. Nuevos conceptos, análisis del desempeño y bases para la acción. Publicación científica y técnica № 589. Washington, D.C.: OPS; 2002.

22. Organización Panamericana de la Salud. Health Situation in the Americas, Basic Indicators, 1997, 1998, 1999, 2000. Washington, D.C.: OPS.

23. Centers for Diseases Prevention and Control, Atlanta, GA, USA. http//:www.cdc.gov.

24. Heise L, Ellsberg M, Gottemoeller M. Ending violence against women. Population Reports, Series L, № 11. Baltimore: JHSPH, Population Information Program; 1999.

25. Walker EA, et al. Adult health status of women with histories of childhood abuse and neglect. Am J Med 1999; 107:332-9.

26. Presidencia de la República del Perú. Legislación sobre violencia familiar. Ministerio de Promoción de la Mujer y del Desarrollo Humano, Unión Europea, Mujer y Ciudadanía. Lima, Perú; 1999.

27. República de Honduras. La Ley contra la violencia doméstica. Instituto Nacional de la Mujer, INAM. Tegucigalpa, Honduras; 1999.

28. Organización Panamericana de la Salud/ Organización Mundial de la Salud. Programa Mujer, Salud y Desarrollo, Centro de Estudios de la Mujer, Honduras. Violencia intrafamiliar. Ruta crítica de las mujeres afectadas. Tegucigalpa, Honduras: OPS; 1999.

29. Organización Panamericana de la Salud/ Organización Mundial de la Salud. Programa Mujer, 
Salud y Desarrollo. Ruta crítica de las mujeres afectadas por la violencia intrafamiliar. San José, Costa Rica: OPS; 1999.

30. Ministerio de Desarrollo Humano. Plan nacional de prevención y erradicación de la violencia contra la mujer. Subsecretaría de Asuntos de Género. La, Paz, Bolivia: OPS; 1994.

31. Ministerio de Salud Pública y Asistencia Social. La Ruta Crítica de las mujeres afectadas por la violencia intrafamiliar en el municipio de Guazapa. El Salvador, San Salvador: OPS/OMS Programa Mujer, Salud y Desarrollo. Mujeres por la dignidad y la vida; (sin fecha).

32. Shrader E, Sagot M. Domestic Violence. Women's way out. Washington, D.C.: Pan American Health Organization; 2000.

33. Ellsberg M, Peña R, Herrera A, Llijestrand J, Winkvist A. Confites en el infierno. Prevalencia y características de la violencia conyugal hacia las mujeres en Nicaragua. Managua, Nicaragua: Red de Mujeres contra la violencia, Universidad de León y UMEA University; 1998.

34. Santacruz M, Concha-Eastman A, Cruz JM. Barrio adentro, la solidaridad violenta de las pandillas. San Salvador, El Salvador. San Salvador, El Salvador: OPS/ OMS, Universidad Centro Americana J.S. Cañas, Instituto Universitario de Opinión Pública, Talleres Gráficos UCA; 2001.

35. Smutt M, Miranda JL. El fenómeno de la violencia en E Salvador. San Salvador, El Salvador: PNUD; 1998.

36. Sanjuán AM. Juventude e violência em Caracas paradoxos de um processo de perda de cidadania. Sâo Paulo sem medo. Río de Janeiro: Garamond Ltda.; 1998. p.155-71.

37. De Mello J M. Adolescentes e jovens como vítimás da violência fatal em São Paulo. En: São Paulo Sem medo. Um diagnóstico da violência urbana. Rio de Janeiro: Editora Garamond; 1998. p.97-120.

38. Moser C, Holland J. Urban poverty and violence in Jamaica. Washington, D.C.: The World Bank Latin American and Caribbean Studies; 1997.

39. Harriot A. Controlling the Jamaican crime problem: peace building and community action. Caribbean Group for Cooperation in Economic Development. West Indies: Department of Government, The University of the West Indies, Mona Campus; 2000.

40. Salazar G. Origen y motivos de la violencia urbana en Santiago y Rancagua (Chile, 1980-1999). En: Bodemer K, Kurtenbach S, Meschkat K, editores. Violencia y regulación de conflictos en América Latina. Asociación Alemana de Investigación sobre América Latina-Adlaf. Caracas: Editorial Nueva Sociedad; 2001. p.91-110.

41. Concha-Eastman A. Pandillas juveniles en América Latina: ¿una alerta social no escuchada? En: Santacruz M, Concha-Eastman A, Cruz JM, editores. Barrio adentro, la solidaridad violenta de las pandillas. San
Salvador, El Salvador: OPS/OMS, Universidad Centro Americana J.S. Cañas, Instituto Universitario de Opinión Pública, Talleres Gráficos UCA; 2001. p.1-21.

42. Carrión F. De la violencia urbana a la seguridad ciudadana. Revista Pretextos (Lima); 1996.

43. Londoño JL, Gaviria A, Guerrero R, editores. Asalto al desarrollo. Violencia en América Latina. Washington, D.C.: Banco Interamericano de Desarrollo, Red de Centros de Investigación; 2000.

44. Morrison A, Orlando MB. Social and economic costs of domestic violence: Chile and Nicaragua. En: Morrisom, Bielh, editors. Too close to home: domestic violence in the Americas. Washington, D.C.: BID; 1999.

45. Concha-Eastman A, Espinosa R, Pineda ME, Melo LM, Becerra ML, Martínez LM, et al. Health sector assistance approach for victims of violence in a developing country. Xth International Symposium on Victimology, Montreal, Canada, Aug. 2000.

46. De Vos E, Goetz MA, Stone DA, Dahlberg LL. Evaluation of a hospital-based youth violence intervention. Am J Prev Med 1996;12:101-8.

47. Olds DL, Henderson Jr. CR, Chamberlin R, Tatelbaum R. Preventing child abuse and neglect: a randomized trial of nurse home visitation. Pediatrics 1986;78:65-78.

48. American Academy of Pediatrics. The role of homevisitation programs in improving health outcomes for children and families. Pediatrics 1998;101:486-9.

49. Roberts I, Kramer MS, Suissa S. Does home visiting prevent childhood injury? A systematic review of randomized controlled trials. BMJ 1996;312:29-33.

50. Olds DL, Eckenrode J, Henderson CR, et al. Longterm effects of home visitation on maternal life course and child abuse and neglect. Fifteen-year follow-up of a randomized trial. JAMA 1997;278:637-43.

51. Ellsberg M, Clavel-Arcas C, Montenegro T, Norori L, Quintanilla M. ¿Cómo atender a las mujeres que viven situaciones de violencia doméstica? Orientaciones básicas para el personal de salud. Red de Mujeres contra la Violencia. Managua, Nicaragua: Editorial INPASA; 1998.

52. Zegarra TM. Redes locales frente a la violencia familiar. Lima, Perú: OPS; 1999.

53. Cruz JM, Portillo PN. Solidaridad y violencia en las pandillas del Gran San Salvador. Más allá de la vida loca. Homies Unidos, Inst. Universitario de Opinión Pública, Radda Barnen de Suecia, Save the Children EU. San Salvador, EI Salvador: UCA Editores; 1998.

54.Organización Panamericana de la Salud/ Organización Mundial de la Salud. Autores varios. Rev Panam Salud Pública 1999;5.

55. Mockus A. Armonizar ley, moral y cultura. Cultura ciudadana, prioridad del gobierno con resultados en prevención y control de violencia, Bogotá, 1995-1997. Documento mimeografiado; 1999. 
56. Alcaldía Mayor de Bogotá, Secretaría de Gobierno. Ciudad y políticas públicas de seguridad y convivencia. Cómo se logró reducir la violencia y la delincuencia en Bogotá, Colombia, 1995-2001. Bogotá: Imprenta Distrital; 2002.

57. Guerrero R, Concha-Eastman A. An Epidemiological approach for the prevention of urban violence: The case of Cali, Colombia. Journal of Health and Population in Developing Countries 2001;4.

58. Krug EG, et al, editors. World report on violence and health. Geneva: World Health Organization; 2002.

59. Ospina W. La decadencia de los dragones. Bogotá: Editorial Alfaguara; 2002. p.7. 